Macedonian Pharmaceutical Bulletin, 66 (Suppl 1) 203 - 204 (2020)

Online ISSN $1857-8969$

UDC: $616.24-002.2-085.458 .03$

DOI: 10.33320/maced.pharm.bull.2020.66.03.101

Short communication

\title{
The impact of clinical pharmacy-led medicines management support for patients with COPD
}

Olivera Krstic Nakovska ${ }^{1}$, Dejan Dokic ${ }^{1}$, Dimitar Karkinski ${ }^{1}$, Sava Pejkovska ${ }^{1}$, Elena Janeva ${ }^{1}$, Sanja Filkova ${ }^{2}$,Zorica Naumovska ${ }^{3}$, Aleksandra Kapedanovska Nestorovska ${ }^{3}$, Ljubica Suturkova ${ }^{3}$

${ }^{1}$ University Clinic of Pulmonology and Allergology, Majka Tereza 17, 1000 Skopje, North Macedonia

${ }^{2}$ Public institution for the needs of University Clinics, Institutes and Urgent Centre,

Department of Clinical Pharmacy, Majka Tereza 17, 1000 Skopje, North Macedonia

${ }^{3}$ Faculty of Pharmacy, Ss. Cyril and Methodius University, Mother Theresa 47, 1000, Skopje, North Macedonia

\section{Introduction}

Chronic obstructive pulmonary disease (COPD) has been defined as a non-fully reversible airflow obstruction, characterised by breathlessness. It is mainly caused by smoking or exposure to pollutants. The goals of COPD treatment are to slow down disease progression, limit symptoms, increase overall health and prevent and treat flare-ups (GOLD, 2020). Inhaled therapies are the mainstay of treatment. Bronchodilatators (short and long acting) have an established role in the management of COPD and significantly reduce the risk of adverse outcomes such as COPD exacerbation. The addition of inhaled corticosteroides may benefit patient who remain symptomatic despite regular treatment with long acting bronchodilatators. The patients should be trained for utilization technique before they are prescribed inhaler by the doctor. Very few published studies focus on adherence in therapy to treatment regimes in COPD, but evidence suggests adherence is poor (Alton and Farndon, 2018). Clinical pharmacist can play an important role in identifying and instruct COPD patients in their inhale techniques to help to improve symptoms burden and medication compliance and overall outcomes (Khdour et al., 2009). The aim of this study is to assess the impact of clinical pharmacy-led management support in patients with COPD in The Clinic of Pulmonology and Allergology of Skopje, Republic of North Macedonia.

\section{Materials and methods}

Observational, longitudinal and prospective study was conducted in The Clinic of Pulmonology and Allergology of Skopje, Republic of North Macedonia. Patients were verbally informed before they were included in the study and 32 eligible hospitalized patients ( 29 men and 2 woman), over 40 years old, with a history of COPD were recruited. Twenty-two patients were assigned in the intervention group and 10 patients were assigned in the usual care or control group. The patients were followed from March 2019 to December 2019 and each of the patients completed COPD assessment test (CAT) and customized Morisky medication adherence scale at baseline, after 3 months and after

\footnotetext{
*okrstik@yahoo.com
} 
6 months. COPD assessment test (CAT) is designed to measure the impact of COPD on a person's life and how this changes over time. It consist of eight simple questions that most patients should be able to understand and answer easily. CAT scores are in to 0-40 range, 0 being very low impact symptoms severity. The customized Moryski adherence scale consists 8 questions that reflect the number of ways medication omission can occur: forgetting, carelessness, stopping when feeling better.

Adherence scores are $0-40$ range with 0 being high adherence and 40 low adherence.Intervention patients were educated on disease state, medication and breathing techniques and they were followed up at 3 and 6 months during a scheduled phone visit.

The intervention of clinical pharmacist on every visit was medication review and follow up as appropriate, reassess inhaler technique, furthur advice and support and fulfill the questionnaries. The patients from the control group received usual hospital inpatient care, from medical and nursing staff, but did not receive the structured intervention by the clinical pharmacist referred above and they fulfilled the questionnaires in 3 and 6 mounts period.

\section{Results and discussion}

The total number of patients that finished the study was 21 in the intervention group and 10 from the control group, because one patient from the intervention group has died during the study. As the study was conducted in the hospitalized patients an overall improvement of tha CAT questionnaire and the customized Morisky adherence scale was confirmed with a significant improvement in the intervention group. In the intervention group the COPD assessment test CAT decreased with a significant difference from $56 \%$ (22.4) at baseline to $22.3 \%$ (8.9) after six months and from $63.5 \%$ (25.4) at baseline to $47.75 \%(19.1)$ at six months in the control group. There was a significant difference between the intervention group and the control group regarding the adherence scale. The confirmed difference was $30.1 \%$ (12.04) vs $21.25 \%$ (8.5) at baseline and $8.75 \%$ (3.5) vs $20.25 \%$ (8.1), after six months in the interventional group in comparison with the control group. The study has confirmed that implementation of the action plan consisting the disease management skills explanation, inhaler technique education led by the clinical pharmacist, was effective and resulted in improved patient satisfaction with improved symptoms management and improved overall outcomes for the intervention group compared with the control group. This was a short time limited study focused on education regarding the disease, inhaler technique and assessment. Adherence results should be treated with caution due to the small patient number and further evaluation is needed in order to assess the impact of this intervention on exacerbation, hospital readmission.

\section{Conclusion}

The clinical pharmacy led intervention can contribute to improve adherence to medication and improve better disease control in COPD patients. Patients have appreciated the time taken by the clinical pharmacist and found face to face contact invaluable, results in improvements in inhaler technique.

\section{References}

Alton, S., Farndon, L., 2018. The impact of community pharmacy-led medicines management support for people with COPD. Br. J. Community Nurs. 23(6), 214-219.

Global strategy for the diagnosis, management, and prevention of chronic obstructive pulmonary disease (2020 report). Available at: https://goldcopd.org/wpcontent/uploads/2019/12/GO LD-2020-FINAL-ver1.2-03Dec19 WMV.pdf.

Khdour, M.R, Kidney, J.C., Smyth, B.M., McElnay, J.C., 2009. Clinical pharmacy-led disease and medicine management programme for patients with COPD. $\mathrm{Br}$. J. Clin. Pharmacol. 68(4), 588-98. 